Département de sciences économiques

9805

\title{
Efficient Strategy-Proof Allocation Functions in Linear Production Economies
}

MANIQUET, François

SPRUMONT, Yves 


\section{Département de sciences économiques}

Université de Montréal

Faculté des arts et des sciences

C.P. 6128, succursale Centre-Ville

Montréal (Québec) H3C 3J7

Canada

http://www.sceco.umontreal.ca

SCECO-information@UMontreal.CA

Téléphone : (514) 343-6539

Télécopieur : (514) 343-7221

Ce cahier a également été publié par le Centre interuniversitaire de recherche en économie quantitative (CIREQ) sous le numéro 0698.

This working paper was also published by the Center for Interuniversity Research in Quantitative Economics (CIREQ), under number 0698.

ISSN 0709-9231 


\title{
CAHIER 9805
}

\section{EFFICIENT STRATEGY-PROOF ALLOCATION FUNCTIONS IN LINEAR PRODUCTION ECONOMIES}

\author{
François MANIQUET ${ }^{1}$ and Yves SPRUMONT ${ }^{2}$
}

1 Fonds National de la Recherche Scientifique and Facultés Universitaires NotreDame de la Paix, Belgium

2 Centre de recherche et développement en économique (C.R.D.E.) and Département de sciences économiques, Université de Montréal

June 1998

The authors thank two anonymous referees for valuable comments. During the preparation of this paper, the first author benefited from the excellent research atmosphere at the Department of Economics and Economic History, Universitat Autónoma de Barcelona, Spain. Partial financial support from HCM grant ERBCHBGCT940699 is gratefully acknowledged. The second author is grateful to the Fonds pour la formation de chercheurs et l'aide à la recherche (FCAR) of Québec and the Social Sciences and Humanities Research Council (SSHRC) of Canada for partial financial support. 


\title{
RÉSUMÉ
}

Dans le cadre d'une économie de production à technologie linéaire, nous caractérisons les règles d'allocation efficaces et non manipulables, tant au sens individuel que coalitionnel. Différentes propriétés de symétries nous permettent ensuite d'isoler une règle unique.

Mots clés : non-manipulabilité, efficacité, ensemble de production linéaire

\begin{abstract}
In a linear production model, we characterize the class of efficient and strategy-proof allocation functions, and the class of efficient and coalition strategy-proof allocation functions. In the former class, requiring equal treatment of equals allows us to identify a unique allocation function. This function is also the unique member of the latter class which satisfies uniform treatment of uniforms.
\end{abstract}

Key words : strategy-proofness, efficiency, linear production set 


\section{Introduction}

A group of agents jointly own a bundle of goods and a linear technology which enables them to transform these goods into one another at constant rates. Preferences are selfish and strictly monotonic. As an example, we can think of an area of homogeneous land which has to be allocated among agents as a function of the purposes (e. g. building, farming) for which they would like to use their plot. It is reasonable to assume that any unit of land used for a given purpose can be transformed into a unit of land used for an other purpose.

An allocation function assigns to each preference profile a feasible bundle of individual consumption bundles. We look for efficient and non manipulable allocation functions.

The non manipulability requirements we are interested in are strategyproofness and coalition strategy-proofness. An allocation function is strategyproof if truthfully reporting one's preferences is a dominant strategy of the direct revelation game associated with the allocation function (that is, the game where the set of admissible strategies is the set of admissible preferences and the outcome associated to a profile of strategies is determined according to the allocation function). An allocation rule is coalition strategyproof if no coalition of agents can ever benefit from jointly misreporting their preferences.

The results are: a characterization of the class of efficient and strategyproof allocation functions, and the class of efficient and coalition strategyproof allocation functions. We also identify the subclasses of these two classes which we obtain when we also impose horizontal equity requirements (which are consequences of the classical anonymity requirement).

It is well known that the requirements of efficiency and strategy-proofness lead to dictatorship in two agent private good economies (cfr. Zhou [9]), and, more generally, conflict with anonymity when there are more than two agents (cfr. Barberà and Jackson [1]). ${ }^{1}$ Strategy-proof allocation functions in production economies without the restriction to a linear technology are analysed in Shenker [7]. ${ }^{2}$ On this larger domain, no efficient, strategy-proof, and horizontally equitable allocation rule exists.

\footnotetext{
${ }^{1}$ The class of economies where a fixed amount of a private commodity has to be divided among agents having single-peaked preferences, however, is an example of a model where strategy-proofness, efficiency, and anonymity do not conflict (see Sprumont [8], Ching [4] and Barberà, Jackson and Neme [2]).

${ }^{2}$ Shenker restricts his attention to allocation functions which satisfy differentiability conditions, thereby excluding almost all of the rules which we identify.
} 
In contrast with the results obtained in these papers, efficiency and strategy-proofness do not conflict with anonymity in our setting. Actually, we identify a unique anonymous allocation function that is efficient and strategy-proof. This allocation function, which could be called the equal budget free choice function, assigns to each agent her preferred bundle in a budget set which is the same for all agents, and whose "slope" is determined by the fixed technological transformation rates.

Our characterization of the equal budget free choice function can be related to the result obtained by Barberà and Jackson [1]. They showed that, in exchange economies, strategy-proofness and anonymity lead to fixed proportion trading away from equal split (given the agents' announced preferences, a fixed trade proportion is chosen out of a small (in particular finite) set of prespecified proportions; agents are then assigned the bundle resulting from trade taking place away from the equal split point and in the chosen proportions, given the uniform rationing process (introduced by Benassy [3])). The equal budget free choice function is very much in the same spirit. Agents may trade at fixed prices which are determined by the technological transformation rates. Because of the linear technology assumption, these prices are equilibrium prices for all preference profiles. This is why efficiency is satisfied. Actually, the Barberà and Jackson [1] model and our model are polar cases of the general model where the set of feasible allocations are determined by a convex technology.

The paper is organized as follows. In section 2, we define the model and give the basic definitions. Then, we study strategy-proofness. In section 3 , we state two technical lemmas, which prove essential for the rest of the inquiry. In particular, Lemma 2 says that any efficient and strategy-proof allocation function assigns to an agent one of her preferred bundles in a budget set. The prices associated with this budget set are determined by the technological transformation rates. The budget itself is independent of the agent's preferences.

In section 4, we characterize the class of efficient and strategy-proof allocation functions, and the only function we obtain when we add the equal treatment of equals requirement. Equal treatment of equals simply says that two agents having the same preferences must end up enjoying the same welfare. It is a clear consequence of the classical anonymity requirement. As announced above, this function assigns to each agent her preferred bundle in a budget set which is the same for all agents, and whose slope is determined by the fixed technological parameters.

In section 5, we characterize the class of efficient and coalition strategyproof allocation functions, and we show that we also come to the equal 
budget free choice function if we merely add the uniform treatment of uniforms requirement. Uniform treatment of uniforms says that if all agents have the same preferences, then they all must end up enjoying the same welfare.

\section{The model}

In this section, we present the model, some terminology, and we give the basic definitions. There are $l$ private goods. There is a set $N$ of $n$ agents whose consumption set is $\mathbb{R}_{+}^{l}$. By using a linear technology, the agents can transform goods into one another, so that the production set can be normalized to $Y=\left\{y \in \mathbb{R}_{+}^{l}: \sum_{h=1}^{l} y_{h} \leq 1\right\}$. A preference is a preordering $R$ on $\mathbb{R}_{+}^{l}$ that is continuous and strictly monotonic, that is, $z>z^{\prime} \Rightarrow z P z^{\prime}{ }^{3}$ The set of all preferences is denoted by $\mathcal{R}_{\mathbf{0}}$.

A preference profile is an element $R_{N}$ of $\mathcal{R}_{0}^{N}$. The list of preferences of members of a coalition $S \subseteq N$ is denoted $R_{S}$. For any $i \in N$, let $R_{-i}$ denote an element of $\mathcal{R}_{0}^{N-\{i\}}$. For any $S \subset N$, let $R_{-S}$ denote an element of $\mathcal{R}_{\mathbf{0}}^{N-S}$. An allocation $z_{N}=\left(z_{1}, \ldots, z_{n}\right) \in \mathbb{R}_{+}^{l \times n}$ is a list of bundles $z_{i}, i \in N$, one per agent. An allocation $z_{N}=\left(z_{1}, \ldots, z_{n}\right)$ is feasible if $\sum_{i=1}^{n} z_{i} \in Y$. Let $Z$ denote the set of feasible allocations. Let $\mathcal{R} \subseteq \mathcal{R}_{\mathbf{0}}$. An allocation function $f: \mathcal{R}^{N} \rightarrow Z$ associates each preference profile in its domain $\mathcal{R}^{N}$ with a feasible allocation. For $R_{N} \in \mathcal{R}^{N}$, let $f_{i}\left(R_{N}\right)$ denote the $i$ th component of $f\left(R_{N}\right)$.

Let $A \subseteq \mathbb{R}_{+}^{l}, R \in \mathcal{R}_{\mathbf{0}}$, be given. Then,

$$
m(R, A)=\left\{z \in \mathbb{R}_{+}^{l}: \forall z^{\prime} \in \mathbb{R}_{+}^{l}, z R z^{\prime}\right\} .
$$

Let $r \in \mathbb{R}_{+}, R \in \mathcal{R}_{\mathbf{0}}$ be given. Then, by a slight abuse of notation, we use $m(R, r)$ to denote

$$
m\left(R,\left\{z \in \mathbb{R}_{+}^{l}: \sum_{h=1}^{l} z_{h} \leq r\right\}\right) .
$$

We will use the two following richness assumptions on a set $\mathcal{R}$ of preferences. ${ }^{4}$

\footnotetext{
${ }^{3}$ Vector inequalities: for all $x, x^{\prime} \in \mathbb{R}_{+}^{l}, x \leq x^{\prime} \Leftrightarrow x_{h} \leq x_{h}^{\prime}, \forall h \in\{1, \ldots, l\}, x<x^{\prime} \Leftrightarrow$ $\left[x \leq x^{\prime}\right.$ and $\left.x \neq x^{\prime}\right], x \ll x^{\prime} \Leftrightarrow x_{h}<x_{h}^{\prime}, \forall h \in\{1, \ldots, l\}$.

${ }^{4}$ A regular monotonic curve $C$ in $\mathbb{R}_{+}^{l}$ is the image of a function $\gamma: \mathbb{R}_{+} \rightarrow \mathbb{R}_{+}^{l}$ which is continuous, monotonic (that is, $r<r^{\prime} \Rightarrow \gamma(r)<\gamma\left(r^{\prime}\right)$ ), unbounded, and such that $\gamma(0)=0$. A preference $R \in \mathcal{R}$ is differentiable if there exists a differentiable function $u: \mathbb{R}_{+}^{l} \rightarrow \mathbb{R}_{+}$such that for all $z, z^{\prime} \in \mathbb{R}_{+}^{l}, z R z^{\prime} \Leftrightarrow u(z) \geq u\left(z^{\prime}\right)$.
} 
(A1) for every regular increasing curve $C$ in $\mathbb{R}_{+}^{l}$, there is some differentiable $R \in \mathcal{R}$ such that $m(R, r) \subset C$ for every $r \in[0,1]$,

(A2) for all $R, R^{\prime} \in \mathcal{R}$ and $r \in \mathbb{R}_{+}$, there is some $R^{\prime \prime} \in \mathcal{R}$ such that $\left(m(R, r) \cup m\left(R^{\prime}, r\right)\right) \subseteq m\left(R^{\prime \prime}, r\right)$.

The set $\mathcal{R}_{\mathbf{0}}$ itself satisfies Assumptions (A1) and (A2). An other example is the set of convex preferences in $\mathcal{R}_{\mathbf{0}}$. The set of quasilinear preferences in $\mathcal{R}_{\mathbf{0}}$ does not satisfy Assumption (A1), whereas the set of strictly convex preferences in $\mathcal{R}_{\mathbf{0}}$ does not satisfy Assumption (A2). Let us note that (A2) requires either flat portions of indifference curves or non-convex preferences.

Now, we define the axioms.

PARETO EFFICIENCY

For every $R_{N}=\left(R_{1}, \ldots, R_{n}\right) \in \mathcal{R}^{N}$, and $z_{N}=\left(z_{1}, \ldots, z_{n}\right) \in Z$,

$$
\left[z_{i} R_{i} f_{i}\left(R_{N}\right), \forall i \in N\right] \Rightarrow\left[z_{i} I_{i} f_{i}\left(R_{N}\right), \forall i \in N\right]
$$

Next, we define two horizontal equity axioms.

EQUAL TREATMENT OF EQUALS

For every $R_{N}=\left(R_{1}, \ldots, R_{n}\right) \in \mathcal{R}^{N}$, and $i, j \in N$,

$$
\left[R_{i}=R_{j}\right] \Rightarrow\left[f_{i}\left(R_{N}\right) I_{i} f_{j}\left(R_{N}\right)\right]
$$

\section{UNIFORM TREATMENT OF UNIFORMS}

For every $R_{N}=\left(R_{1}, \ldots, R_{n}\right) \in \mathcal{R}^{N}$,

$$
\left[R_{i}=R_{j}, \forall i, j \in N\right] \Rightarrow\left[f_{i}\left(R_{N}\right) I_{i} f_{j}\left(R_{N}\right), \forall i, j \in N\right]
$$

Observe that uniform treatment of uniforms is logically weaker than equal treatment of equals, and that both axioms are logically weaker than the classical anonymity axiom. Finally, we define two non-manipulability axioms. We distinguish between the case where only individual agents can try to manipulate the outcome (strategy-proofness) and the case where any coalition can try to manipulate the outcome (coalition strategy-proofness).

\section{STRATEGY-PROOFNESS}

For every $R_{N}=\left(R_{1}, \ldots, R_{n}\right) \in \mathcal{R}^{N}, i \in N$ and $R_{i}^{\prime} \in \mathcal{R}$,

$$
f_{i}\left(R_{N}\right) R_{i} f_{i}\left(R_{i}^{\prime}, R_{-i}\right)
$$

COALITION STRATEGY-PROOFNESS

For every $R_{N}=\left(R_{1}, \ldots, R_{n}\right) \in \mathcal{R}^{N}, S \subseteq N$ and $R_{S}^{\prime} \in \mathcal{R}^{S}$,

$$
\left[f_{i}\left(R_{S}^{\prime}, R_{-S}\right) R_{i} f_{i}\left(R_{N}\right), \forall i \in S\right] \Rightarrow\left[f_{i}\left(R_{S}^{\prime}, R_{-S}\right) I_{i} f_{i}\left(R_{N}\right), \forall i \in S\right]
$$




\section{$3 \quad$ Preliminary results}

In this section, we state and prove two preliminary, rather technical, lemmas. The first lemma identifies a general property of strategy-proof allocation functions. The second lemma states that an efficient and strategy-proof allocation function assigns to each agent $i$ her preferred bundle in a budget set $\left\{z \in \mathbb{R}_{+}^{l}: \sum_{h=1}^{l} z_{h}=a_{i}\right\}$ where $a_{i}$, which can be interpreted as agent $i$ 's income, does not depend on her preference, for every $i \in N$.

Lemma 1 Let $\mathcal{R} \subseteq \mathcal{R}_{\mathbf{0}}$. An allocation function $f: \mathcal{R}^{N} \rightarrow Z$ satisfies strategy-proofness if and only if for every $i \in N$, there exists a correspondence $X_{i}: \mathcal{R}^{N-\{i\}} \rightarrow \mathbb{R}_{+}^{l}$, such that for every $R_{N} \in \mathcal{R}^{N}$,

1. $f_{i}\left(R_{i}, R_{-i}\right) \in m\left(R_{i}, X_{i}\left(R_{-i}\right)\right)$,

2. if $x, x^{\prime} \in X_{i}\left(R_{-i}\right)$, then $x \leq x^{\prime} \Rightarrow x=x^{\prime}$.

Proof: The straightforward "if" part is omitted. Now, let us suppose that $f$ is strategy-proof. For every $i \in N, R_{-i} \in \mathcal{R}^{N-\{i\}}$, we can define the option set $O_{i}\left(R_{-i}\right) \subset \mathbb{R}_{+}^{l}$ by

$$
O_{i}\left(R_{-i}\right)=\left\{z \in \mathbb{R}_{+}^{l}: \exists R_{i} \in \mathcal{R}: z=f_{i}\left(R_{i}, R_{-i}\right)\right\}
$$

By strategy-proofness, $f_{i}\left(R_{i}, R_{-i}\right) \in m\left(R_{i}, O_{i}\left(R_{-i}\right)\right)$. By strict monotonicity of the preferences and strategy-proofness, no two elements of $O_{i}\left(R_{-i}\right)$ are ordered: if $x, x^{\prime} \in O_{i}\left(R_{-i}\right)$, then $x \leq x^{\prime} \Rightarrow x=x^{\prime}$. Therefore, we can define $X_{i}$ by associating every $R_{-i}$ with the corresponding $O_{i}\left(R_{-i}\right)$. Q.E.D.

Lemma $2{ }^{5}$ Let $\mathcal{R} \subseteq \mathcal{R}_{\mathbf{0}}$ satisfy Assumption (A1). An allocation function $f: \mathcal{R}^{N} \rightarrow Z$ satisfies strategy-proofness and efficiency only if for every $i \in N$, there exists $a_{i}: \mathcal{R}^{N-\{i\}} \rightarrow \mathbb{R}_{+}$, such that for every $R_{N} \in \mathcal{R}^{N}$,

$$
f_{i}\left(R_{i}, R_{-i}\right) \in m\left(R_{i}, a_{i}\left(R_{-i}\right)\right)
$$

Proof: Fix an arbitrary agent $i \in N$, and a profile $R_{-i} \in \mathcal{R}^{N-\{i\}}$. Note that if $X_{i}\left(R_{-i}\right)=\{(0, \ldots, 0)\}$, then we are done. So we assume that it is not the case. We begin by noting a straightforward consequence of efficiency: for every regular increasing curve $C$ and corresponding $R \in \mathcal{R}$ satisfying the properties of Assumption (A1), $f_{i}\left(R, R_{-i}\right) \in C$. Indeed, suppose $f_{i}\left(R, R_{-i}\right) \notin C$.

\footnotetext{
${ }^{5}$ We are grateful to José Wuidar for his help with the proof of this lemma.
} 
Let $r=\sum_{h} f_{i}^{h}\left(R, R_{-i}\right)$. Since $m(R, r) \subset C, f_{i}\left(R, R_{-i}\right) \notin m(R, r)$. Pick $x_{i} \in m(R, r)$. Then,

$$
\begin{aligned}
& \text { i) } \quad \sum_{h} x_{i}^{h}=\sum_{h} f_{i}^{h}\left(R, R_{-i}\right)=r \text {, and } \\
& \text { ii) } \quad x_{i} P_{i} f_{i}^{h}\left(R, R_{-i}\right) .
\end{aligned}
$$

But then $\left(x_{i}, f_{-i}\left(R, R_{-i}\right)\right)$ Pareto dominates $f\left(R, R_{-i}\right)$. We continue the proof by the following claim.

Claim 1: for every regular increasing curve $C$ in $\mathbb{R}_{+}^{l}, C \cap X_{i}\left(R_{-i}\right) \neq \emptyset$. Suppose not. By Assumption (A1), there exists $R \in \mathcal{R}$ such that for all $r \in[0,1]$, $m(R, r) \subset C$. By Lemma 1 and strategy-proofness, $f_{i}\left(R, R_{-i}\right) \in X_{i}\left(R_{-i}\right)$, so that $f_{i}\left(R, R_{-i}\right) \notin C$, which contradicts efficiency.

Now, if the statement of the Lemma is not true, there exist $R_{i}, R_{i}^{\prime} \in \mathcal{R}$, $z_{i}, z_{i}^{\prime} \in \mathbb{R}_{+}^{l}$ such that $f_{i}\left(R_{i}, R_{-i}\right)=z_{i}, f_{i}\left(R_{i}^{\prime}, R_{-i}\right)=z_{i}^{\prime}$, and $\sum_{h=1}^{l} z_{i h} \neq$ $\sum_{h=1}^{l} z_{i h}^{\prime}$. Let the parameters $z_{i}, z_{i}^{\prime}, R_{i}, R_{i}^{\prime}$ be fixed for the rest of the proof. We fix

$$
a=\sum_{h=1}^{l} z_{i h} .
$$

By claim 1 , there exists $\bar{\lambda}_{1} \in \mathbb{R}_{+}$such that $\left(0, \bar{\lambda}_{1} \frac{z_{i 2}}{\sum_{h \neq 1} z_{i h}}, \ldots, \bar{\lambda}_{1} \frac{z_{i l}}{\sum_{h \neq 1} z_{i h}}\right)$ $\in X_{i}\left(R_{-i}\right)$. By Lemma 1 proviso 2, this number is unique. Define $g_{1}$ : $\left[0, \bar{\lambda}_{1}\right] \rightarrow R_{+}$by

$$
g_{1}(\lambda)=y \Leftrightarrow\left(y, \lambda \frac{z_{i 2}}{\sum_{h \neq 1} z_{i h}}, \ldots, \lambda \frac{z_{i l}}{\sum_{h \neq 1} z_{i h}}\right) \in X_{i}\left(R_{-i}\right)
$$

so that

$$
g_{1}\left(\sum_{h \neq 1} z_{i h}\right)=z_{i 1}
$$

By claim 1 and Lemma 1 proviso 2, $g_{1}$ is a well-defined, bounded, strictly decreasing, continuous function.

Consequently, by Lebesgue's theorem, $g_{1}$ is differentiable almost everywhere in $\left[0, \bar{\lambda}_{1}\right], g_{1}^{\prime}$ is integrable, and

$$
\int_{0}^{\bar{\lambda}_{1}} g_{1}^{\prime}(\lambda) d \lambda \geq g_{1}\left(\bar{\lambda}_{1}\right)-g_{1}(0)
$$


By efficiency and claim $1, g_{1}^{\prime}(\lambda)=c=-1$ whenever $g_{1}^{\prime}(\lambda)$ exists, where -1 is the value of the projection on that hyperplane of the directional derivative of some differentiable preferences associated to a curve $C$ intersecting $X_{i}\left(R_{-i}\right)$ at $\left(g_{1}(\lambda), \lambda \frac{z_{i 2}}{\sum_{h \neq 1} z_{i h}}, \ldots, \lambda \frac{z_{i l}}{\sum_{h \neq 1} z_{i h}}\right)$. Now consider the inverse function $g_{1}^{-1}$. Exactly as above, claim 1 and Lemma 1 proviso 2 imply that $g_{1}^{-1}$ is differentiable almost everywhere in $\left[0, g_{1}(0)\right], g_{1}^{-1 \prime}$ is integrable, and

$$
\int_{0}^{g_{1}(0)} g_{1}^{-1 \prime}(y) d y \geq g_{1}^{-1}\left(g_{1}(0)\right)-g_{1}^{-1}(0)
$$

Also, by efficiency and claim $1, g_{1}^{-1 \prime}(y)=\frac{1}{c}=-1$ whenever $g_{1}^{-1 \prime}(y)$ exists. From equation (3), we derive

$$
c \bar{\lambda}_{1} \geq-g_{1}(0)
$$

From equation (4), we derive

$$
\frac{1}{c} g_{1}(0) \geq-\bar{\lambda}_{1}
$$

Putting equations (5) and (6) together, we get $c=\frac{-g_{1}(0)}{\bar{\lambda}_{1}}$. Now, using equations (1) and (2), we get

$$
g_{1}(\lambda)=a-\lambda
$$

We now claim that $z_{i 1}^{\prime}<a$. Suppose not. By the preceding claim, $(a, 0, \ldots, 0) \in X_{i}\left(R_{-i}\right)$. Let $R_{i}^{\star} \in \mathcal{R}$ be such that $f_{i}\left(R_{i}^{\star}, R_{-i}\right)=(a, 0, \ldots, 0)$. By strict monotonicity, $z_{i}^{\prime} P_{i}^{\star}(a, 0, \ldots, 0)$, contradicting strategy-proofness. Let $g_{2}: \mathbb{R}_{+} \rightarrow \mathbb{R}_{+}$be defined by $g_{2}(\lambda)=y \Leftrightarrow\left(z_{i 1}^{\prime}, y, \lambda \frac{z_{i 3}}{\sum_{h \neq 1,2} z_{i h}}, \ldots, \lambda \frac{z_{i l}}{\sum_{h \neq 1,2} z_{i h}}\right)$ $\in X_{i}\left(R_{-i}\right)$. Following the same arguments as above, we get $g_{2}(\lambda)=(a-$ $\left.z_{i 1}^{\prime}\right)-\lambda$. By replicating the arguments $l-1$ times, we prove that $\left(z_{i 1}^{\prime}, z_{i 2}^{\prime}, \ldots\right.$, $\left.z_{i l-1}, a-\sum_{h=1}^{l-1} z_{i h}^{\prime}\right) \in X_{i}\left(R_{-i}\right)$ which, by strict monotonicity of the preferences and strategy-proofness of $f$ is incompatible with $z_{i}^{\prime} \in X_{i}\left(R_{-i}\right)$. Q.E.D.

\section{Strategy-proofness}

We are now equipped to state our first theorem, which provides a complete characterization of efficient and strategy-proof allocation functions. The lesson is that when agents are not able to form coalitions, efficiency and strategy-proofness do not prevent a planner from taking all preferences into account when allocating the goods. 
Theorem 1 Let $\mathcal{R} \subseteq \mathcal{R}_{\mathbf{0}}$ satisfy Assumption (A1). An allocation function $f: \mathcal{R}^{N} \rightarrow Z$ satisfies strategy-proofness and efficiency if and only if for every $i \in N$, every coalition $S \subset N$ of cardinality $n-2$ not containing $i$, there is a mapping $b_{i S}: \mathcal{R}^{S} \rightarrow \mathbb{R}_{+}$such that for every $R_{N} \in \mathcal{R}^{N}$,

- $\sum_{S: i \notin S,|S|=n-2} b_{i S}\left(R_{S}\right) \geq 0, \forall i \in N$

- $\sum_{i \in N} \sum_{S: i \notin S,|S|=n-2} b_{i S}\left(R_{S}\right)=1$, and

- $f_{i}\left(R_{N}\right) \in m\left(R_{i}, \sum_{S: i \notin S,|S|=n-2} b_{i S}\left(R_{S}\right)\right), \forall i \in N$.

Before proving Theorem 1, it may be useful to illustrate it in the threeagent case. In that case, $\{S: 1 \notin S,|S|=n-2\}=\{\{2\},\{3\}\},\{S: 2 \notin$ $S,|S|=n-2\}=\{\{1\},\{3\}\}$, and $\{S: 3 \notin S,|S|=n-2\}=\{\{1\},\{2\}\}$. Thus, if $f$ is strategy-proof and efficient, there exist mappings $b_{12}, b_{13}, b_{21}$, $b_{23}, b_{31}$, and $b_{32}$, such that for any profile $\left(R_{1}, R_{2}, R_{3}\right)$,

$$
\left.\begin{array}{l}
f_{1}\left(R_{1}, R_{2}, R_{3}\right) \in m\left(R_{1}, b_{12}\left(R_{2}\right)+b_{13}\left(R_{3}\right)\right) \\
f_{2}\left(R_{1}, R_{2}, R_{3}\right) \in m\left(R_{2}, b_{21}\left(R_{1}\right)+b_{23}\left(R_{3}\right)\right) \\
f_{3}\left(R_{1}, R_{2}, R_{3}\right) \in m\left(R_{3}, b_{31}\left(R_{1}\right)+b_{32}\left(R_{2}\right)\right)
\end{array}\right\}
$$

Inequalities $\sum_{j \neq i} b_{i j}\left(R_{j}\right) \geq 0$ guarantee that each agent's consumption lies in the consumption set and the constraint $\sum_{i} \sum_{j \neq i} b_{i j}\left(R_{j}\right)=1$ ensures that the aggregate consumption is in the production set. Equation (7) means that each agent is maximizing her preference in a budget set that is determined by the other agents' preferences. Notice that these preferences affect the budget in an additively separable way. According to Theorem 1, a weak form of additive separability persists when there are more than three agents. ${ }^{6}$

Proof of Theorem 1: The proof of the straightforward "if" part is omitted. Let $f: \mathcal{R}^{N} \rightarrow Z$ be an strategy-proof and efficient allocation function. For each $i \in N$, there exists, by Lemma 2 , a mapping $a_{i}: \mathcal{R}^{N \backslash\{i\}} \rightarrow \mathbb{R}_{+}$such that for every $R_{N} \in \mathcal{R}^{N}, f_{i}\left(R_{N}\right) \in m\left(R_{i}, a_{i}\left(R_{-i}\right)\right)$ for all $i \in N$ and

$$
\sum_{i \in N} a_{i}\left(R_{-i}\right)=1
$$

Fix now an arbitrary $j \in N$, say, $j=1$. We first claim that the mapping $a_{1}$ is additively separable in the sense that

$$
a_{1}\left(R_{-1}\right)=\sum_{S: 1 \notin S,|S| \leq n-2} a_{1 S}\left(R_{S}\right), \quad \forall R_{-1} \in \mathcal{R}^{N \backslash\{1\}}
$$

\footnotetext{
${ }^{6}$ Equation (7) can be further simplified by noting that $b_{12}=-b_{32}, b_{13}=-b_{23}$ and $b_{21}=-b_{31}$. Such obvious simplifications are not available for more than three agents.
} 
for properly chosen mappings $a_{1 S}$, where we interpret $a_{1 \emptyset}$ as a constant.

To prove this claim, fix an arbitrary profile $R_{-1} \in \mathcal{R}^{N \backslash\{1\}}$. Let $R^{0}$ be an arbitrary preference in $\mathcal{R}$ which is distinct from $R_{i}$ for all $i \in N \backslash\{1\}$. Consider now the set $\mathcal{R}\left(R^{0}, R_{-1}\right)$ of all preference profiles $R_{N}^{\prime} \in \mathcal{R}^{N}$ for which $R_{1}^{\prime}=R^{0}$ and $R_{i}^{\prime} \in\left\{R_{i}, R^{0}\right\}$, for all $i \in N \backslash\{1\}$. For each $i \in N \backslash\{1\}$ and $R_{N}^{\prime} \in \mathcal{R}\left(R^{0}, R_{-1}\right)$, define

$$
t_{i}\left(R_{N}^{\prime}\right)=\left\{\begin{array}{lll}
1 & \text { if } & R_{i}^{\prime}=R_{i} \\
2 & \text { if } & R_{i}^{\prime}=R^{0}
\end{array}\right.
$$

and let $t\left(R_{N}^{\prime}\right)=\sum_{i \in N \backslash\{1\}} t_{i}\left(R_{N}^{\prime}\right)$. Since the number of profiles $R_{N}^{\prime} \in$ $\mathcal{R}\left(R^{0}, R_{-1}\right)$ for which $t\left(R_{N}^{\prime}\right)$ is odd equals the number of those for which it is even, we know from (8) that

$$
\sum_{R_{N}^{\prime} \in \mathcal{R}\left(R^{0}, R_{-1}\right)}\left((-1)^{t\left(R_{N}^{\prime}\right)+n-1} \sum_{i \in N} a_{i}\left(R_{-i}^{\prime}\right)\right)=0
$$

But for each $i \in N \backslash\{1\}, \sum_{R_{N}^{\prime} \in \mathcal{R}\left(R^{0}, R_{-1}\right)}(-1)^{t\left(R_{N}^{\prime}\right)+n-1} a_{i}\left(R_{-i}^{\prime}\right)=0$ (since each $a_{i}\left(R_{-i}^{\prime}\right)$ is generated by both $\left(R_{i}, R_{-i}^{\prime}\right)$ and $\left(R^{0}, R_{-i}^{\prime}\right)$ and thus appears once with a +1 coefficient, and once with a -1 coefficient). Therefore,

$$
\sum_{R_{N}^{\prime} \in \mathcal{R}\left(R^{0}, R_{-1}\right)}(-1)^{t\left(R_{N}^{\prime}\right)+n-1} a_{1}\left(R_{-1}^{\prime}\right)=0
$$

which can be rewritten as

$$
a_{1}\left(R_{-1}\right)=\sum_{R_{N}^{\prime} \in \mathcal{R}\left(R^{0}, R_{-1}\right) \backslash\left\{\left(R^{0}, R_{-1}\right)\right\}}(-1)^{t\left(R_{N}^{\prime}\right)+n} a_{1}\left(R_{-1}^{\prime}\right)
$$

Now, we can define a one-to-one correspondence between the profiles in $\mathcal{R}\left(R^{0}, R_{-1}\right)$ and the subsets of $N \backslash\{1\}$ by associating $R_{N}^{\prime}$ with the coalition $S$ of those agents $i$ for whom $R_{i}^{\prime}=R_{i}$. Observe that $t\left(R_{N}^{\prime}\right)=2(n-1)-|S|$, which means that $t\left(R_{N}^{\prime}\right)+n$ is odd if and only if $n-|S|$ is odd. Thus we obtain

$$
a_{1}\left(R_{-1}\right)=\sum_{S: 1 \notin S,|S| \leq n-2}(-1)^{n-|S|} a_{1}\left(R_{S}, R^{0}, \ldots, R^{0}\right)
$$

where the preference $R^{0}$ is held by all members of $N \backslash(S \cup\{i\})$. Defining $a_{1 S}\left(R_{S}\right)=(-1)^{n-|S|} a_{1}\left(R_{S}, R^{0}, \ldots, R^{0}\right)$ completes the proof of the claim.

We have now to derive the $b_{1 S}$ mappings from the above $a_{1 S}$ mappings. Index and rank the coalitions of size $n-2$ not containing $i$ (there are $n-1$ 
of them) according to the lexicographic ordering derived from the natural ordering of the agents: ${ }^{7}$

$$
S_{1} \prec S_{2} \prec \ldots \prec S_{n-1}
$$

For each $k, 1 \leq k \leq n-1$, and each $R_{S_{k}} \in \mathcal{R}^{S_{k}}$, let

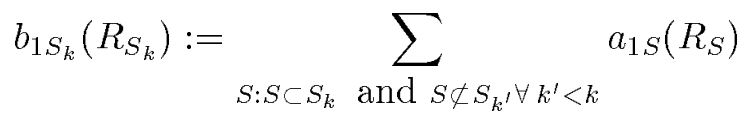

The proof is now complete since the choice of agent 1 was arbitrary. Q.E.D.

Note that Theorem 1 does not assert - and it is generally false - that the system of $b_{i S}$ mappings is unique. On the other hand, it may seem curious that the $b_{i S}$ mappings must be defined for coalitions containing as many as $n-2$ agents. It is impossible, however, to restrict ourselves to $b_{i S}$ defined with respect to strictly smaller coalitions, as examplified by the following function. Let $I($.$) denote the indicator function. Let \mathcal{R}^{*} \subset \mathcal{R}^{0}$. Let $f: \mathcal{R}^{0 N} \rightarrow Z$ be defined by:

$$
\begin{aligned}
& b_{1, N \backslash\{1,2\}}=\frac{1}{n}+\frac{1}{n} I\left(R_{3} \in \mathcal{R}^{*}, \ldots, R_{n} \in \mathcal{R}^{*}\right) \\
& b_{2, N \backslash\{1,2\}}=\frac{1}{n}-\frac{1}{n} I\left(R_{3} \in \mathcal{R}^{*}, \ldots, R_{n} \in \mathcal{R}^{*}\right) \\
& \forall S \subset N, 1 \notin S,|S|=n-2 \text { and } S \neq N \backslash\{1,2\} \quad: \quad b_{1 S} \equiv 0 \\
& \forall S \subset N, 2 \notin S,|S|=n-2 \text { and } S \neq N \backslash\{1,2\} \quad: \quad b_{2 S} \equiv 0
\end{aligned}
$$

and for all $j \in N \backslash\{1,2\}, \sum_{S} b_{j S} \equiv \frac{1}{n}$. Finally, note that if there exist a regular increasing curve $C$ in $\mathbb{R}_{+}^{l}$, and preferences $R, R^{\prime} \in \mathcal{R}^{0}$ such that $m(R, r) \subset C$ and $m\left(R^{\prime}, r\right) \subset C$ for every $r \in[0,1]$, and $R \in \mathcal{R}^{*}$ whereas $R^{\prime} \notin \mathcal{R}^{*}$, then $f$ is bossy. ${ }^{8}$

Our next result is that if equal treatment of equals is added to efficiency and strategy-proofness, then all budgets must be constant and equal.

\footnotetext{
${ }^{7}$ The ordering $\prec$ is formally defined as follows. Rewrite each coalition $S=$ $\left\{i_{1}(S), \ldots, i_{n-2}(S)\right\}$ so that $i_{1}(S)<\ldots<i_{n-2}(S)$. Then $S \prec T$ if there exists $k$, $1 \leq k \leq n-2$, such that $i_{k}(S)=i_{k}(T)$ and $i_{k^{\prime}}(S)<i_{k^{\prime}}(T)$ whenever $1 \leq k^{\prime}<k$.

${ }^{8}$ Recall that a function is bossy if there exist $i, j \in N, R_{N} \in \mathcal{R}^{N}$ and $\bar{R}_{i}^{\prime} \in \mathcal{R}$ such that $f_{i}\left(R_{N}\right)=f_{i}\left(R_{i}^{\prime}, R_{-i}\right)$ whereas $f_{j}\left(R_{N}\right)=f_{j}\left(R_{i}^{\prime}, R_{-i}\right)$.
} 
Theorem 2 Let $\mathcal{R} \subseteq \mathcal{R}_{\mathbf{0}}$ satisfy Assumption (A1). An allocation function $f: \mathcal{R}^{N} \rightarrow Z$ satisfies strategy-proofness, efficiency, and equal treatment of equals if and only if for every $R_{N} \in \mathcal{R}^{N}, i \in N$,

$$
f_{i}\left(R_{N}\right) \in m\left(R_{i}, \frac{1}{n}\right)
$$

Proof: The proof of the straightforward "if" part is omitted.

Let the allocation function $f$ satisfy the three axioms. Let again $a_{1}, \ldots, a_{n}$ be the mappings identified in Lemma 2 . We must show that

$$
a_{i}\left(R_{-i}\right)=\frac{1}{n}, \forall i \in N, \forall R_{N} \in \mathcal{R}^{N}
$$

Fix an arbitrary preference $R^{0}$. Since equal treatment of equals requires that $f_{i}\left(R^{0}, \ldots, R^{0}\right) I^{0} f_{j}\left(R^{0}, \ldots, R^{0}\right)$ for all $i, j \in N$ and since $R^{0}$ is strictly monotonic, $a_{i}\left(R^{0}, \ldots, R^{0}\right)=a_{j}\left(R^{0}, \ldots, R^{0}\right)$, for all $i, j \in N$. Using (8), we obtain

$$
a_{i}\left(R^{0}, \ldots, R^{0}\right)=\frac{1}{n}, \forall i \in N
$$

We complete the proof by an induction argument on the number of preferences differing from $R^{\mathbf{0}}$. For each $R_{N} \in \mathcal{R}^{N}$, let $S^{\mathbf{0}}\left(R_{N}\right)=\left\{i \in N: R_{i}=\right.$ $\left.R^{0}\right\}$, and $S\left(R_{N}\right)=N \backslash S^{0}\left(R_{N}\right)$, and let $s\left(R_{N}\right)$ be the cardinality of the latter set. We have just shown that equation (9) holds if $s\left(R_{N}\right)=0$. We now fix $\sigma, 1 \leq \sigma \leq n$, and assume that (9) holds whenever $s\left(R_{N}\right) \leq \sigma-1$. We pick a profile $R_{N}$ for which $s\left(R_{N}\right)=\sigma$, and prove (9) for that profile.

For each $i \in S\left(R_{N}\right)$, construct the profile $\left(R^{0}, R_{-i}\right)$ by replacing $R_{i}$ with $R^{0}$ and observe that $s\left(R^{0}, R_{-i}\right)=\sigma-1$. The induction hypothesis thus yields that

$$
a_{i}\left(R_{-i}\right)=\frac{1}{n} \forall i \in S\left(R_{N}\right)
$$

Taking (8) into account, we get

$$
\sum_{i \in S^{\circ}\left(R_{N}\right)} a_{i}\left(R_{-i}\right)=1-\frac{\sigma}{n}
$$

By equal treatment of equals,

$$
a_{i}\left(R_{-i}\right)=\frac{1}{n-\sigma}\left(1-\frac{\sigma}{n}\right)=\frac{1}{n}, \forall i \in S^{\mathbf{0}}\left(R_{N}\right)
$$

and the proof is complete. Q.E.D. 


\section{Coalition strategy-proofness}

In this section, we assume that the agents have the opportunity to coordinate their misreports. If the preference domain is rich enough, coalition strategyproofness and efficiency force us to give each agent his best choice in a fixed budget set.

Theorem 3 Let $\mathcal{R} \subseteq \mathcal{R}_{\mathbf{0}}$ satisfy Assumptions (A1) and (A2). An allocation function $f: \mathcal{R}^{N} \rightarrow Z$ satisfies coalition strategy-proofness and efficiency if and only if there exists $\alpha_{N}=\left(\alpha_{1}, \ldots, \alpha_{n}\right) \in \mathbb{R}_{+}^{n}$ such that for every $R_{N} \in \mathcal{R}^{N}$,

- $\sum_{i=1}^{n} \alpha_{i}=1$, and

- $f_{i}\left(R_{N}\right) \in m\left(R_{i}, \alpha_{i}\right), \forall i \in N$.

Proof: Here again, we leave the easy proof of the "if" part to the reader.

Fix a preference domain $\mathcal{R}$ satisfying Assumptions (A1) and (A2) and an efficient and coalition strategy-proof allocation function $f$ on $\mathcal{R}^{N}$. For each $i \in N$, there is a mapping $a_{i}$ such that $f_{i}\left(R_{N}\right) \in m\left(R_{i}, a_{i}\left(R_{-i}\right)\right)$ for each $R_{N}$, and equation (8) holds for each $R_{N}$.

Choose a preference $R^{0} \in \mathcal{R}$ and set $\alpha_{i}=a_{i}\left(R^{0}, \ldots, R^{0}\right)$ for all $i \in N$. We claim that $a_{i}\left(R_{-i}\right)=\alpha_{i}$ for all $R_{N} \in \mathcal{R}^{N}$ and all $i \in N$. Since $R_{-i}$ can be transformed into $\left(R^{0}, \ldots, R^{0}\right)$ by successively replacing each component, we need only prove that

$$
a_{i}\left(R_{-i}\right)=a_{i}\left(R_{-i j}, R_{j}^{\prime}\right), \forall i \in N \backslash\{j\}, \forall j \in N, \forall R_{N} \in \mathcal{R}^{N}, \forall R_{j}^{\prime} \in \mathcal{R}
$$

Fix $j, R_{N}$ and $R_{j}^{\prime}$ for the rest of the proof. Thanks to Assumption (A2), there exists some $R_{j}^{\prime \prime} \in \mathcal{R}$ such that $\left(m\left(R_{j}, a_{j}\left(R_{-j}\right)\right) \cup m\left(R_{j}^{\prime}, a_{j}\left(R_{-j}\right)\right)\right) \subseteq$ $m\left(R_{j}^{\prime \prime}, a_{j}\left(R_{-j}\right)\right)$. By definition, $f_{j}\left(R_{j}^{\prime \prime}, R_{-j}\right) I_{j}^{\prime \prime} f_{j}\left(R_{N}\right) I_{j}^{\prime \prime} f_{j}\left(R_{j}^{\prime}, R_{-j}\right)$. But then, coalition strategy-proofness requires

$$
f_{i}\left(R_{j}^{\prime \prime}, R_{-j}\right) R_{i} f_{i}\left(R_{N}\right), \forall i \in N \backslash\{j\}
$$

and

$$
f_{i}\left(R_{j}^{\prime \prime}, R_{-j}\right) R_{i} f_{i}\left(R_{j}^{\prime}, R_{-j}\right), \forall i \in N \backslash\{j\}
$$

Indeed, if (11) is violated for some $i \neq j$, then coalition $\{i, j\}$ manipulates at profile $\left(R_{j}^{\prime \prime}, R_{-j}\right)$ by announcing $\left(R_{i}, R_{j}\right)$. Likewise, if $(12)$ is violated for some $i \neq j$, then coalition $\{i, j\}$ manipulates at profile $\left(R_{j}^{\prime \prime}, R_{-j}\right)$ by announcing $\left(R_{i}, R_{j}^{\prime}\right)$. 
Since $f_{i}\left(R_{j}^{\prime \prime}, R_{-j}\right) \in m\left(R_{i}, a_{i}\left(R_{-i j}, R_{j}^{\prime \prime}\right)\right)$ and $f_{i}\left(R_{N}\right) \in m\left(R_{i}, a_{i}\left(R_{-i}\right)\right)$, it follows from (11) that

$$
a_{i}\left(R_{-i j}, R_{j}^{\prime \prime}\right) \geq a_{i}\left(R_{-i}\right), \forall i \in N \backslash\{j\}
$$

because $R_{i}$ is strictly monotonic. Likewise, (12) yields

$$
a_{i}\left(R_{-i j}, R_{j}^{\prime \prime}\right) \geq a_{i}\left(R_{-i j}, R_{j}^{\prime}\right), \forall i \in N \backslash\{j\}
$$

Since $a_{j}\left(R_{-j}\right)+\sum_{i \neq j} a_{i}\left(R_{-i j}, R_{j}^{\prime \prime}\right)=1=a_{j}\left(R_{-j}\right)+\sum_{i \neq j} a_{i}\left(R_{-i}\right)$, all inequalities in (13) and (14) must be equalities. Since we have chosen $j, R_{N}$, and $R_{j}^{\prime}$ arbitrarily, equation (10) follows. Q.E.D.

Assumption (A2) in Theorem 3 cannot be dropped. To see this, consider the following 3 -agent, 2 -good example. The domain $\mathcal{R}$ consists of the continuous, strictly convex, strictly monotonic preferences. For each $R \in \mathcal{R}$, let $a(R) \in\left[0, \frac{1}{3}\right]$ be the first coordinate of the unique element of $m\left(R, \frac{1}{3}\right)$. For each $R_{N} \in \mathcal{R}^{N}$, let

$$
\begin{aligned}
& f_{1}\left(R_{N}\right)=m\left(R_{1}, \frac{1}{3}\right) \\
& f_{2}\left(R_{N}\right)=m\left(R_{2}, \frac{1}{3}+a\left(R_{1}\right)\right) \\
& f_{3}\left(R_{N}\right)=m\left(R_{3}, \frac{1}{3}-a\left(R_{1}\right)\right)
\end{aligned}
$$

This rule is efficient and coalition strategy-proof. ${ }^{9}$

An immediate consequence of Theorem 3 is

Theorem 4 Let $\mathcal{R} \subseteq \mathcal{R}_{\mathbf{0}}$ satisfy Assumptions (A1) and (A2). The allocation function $f: \mathcal{R}^{N} \rightarrow Z$ satisfies coalition strategy-proofness, efficiency, and uniform treatment of uniforms if and only if $f_{i}\left(R_{N}\right) \in m\left(R_{i}, \frac{1}{n}\right)$ for all $i \in N$ and $R_{N} \in \mathcal{R}^{N}$.

An alternative characterization of the equal budget free choice function was given in Theorem 2. A simple three-agent example shows that coalition strategy-proofness cannot be replaced with strategy-proofness in Theorem

\footnotetext{
${ }^{9}$ As we mentioned above, the set of strictly convex preferences does not satisfy assumption (A2). We have not been able to characterize the class of coalition strategy-proof and efficient functions defined over the domain of strictly convex preferences.
} 
4. Partition $\mathcal{R}$ in two arbitrary subsets $\mathcal{R}^{+}$and $\mathcal{R}^{-}$, and let each agent maximize her preference in the budget sets

$$
\begin{aligned}
& a_{1}\left(R_{2}, R_{3}\right)=\left\{\begin{array}{l}
\frac{1}{2} \text { if }\left(R_{2}, R_{3}\right) \in\left(\mathcal{R}^{-} \times \mathcal{R}^{+}\right), \\
\frac{1}{6} \text { if }\left(R_{2}, R_{3}\right) \in\left(\mathcal{R}^{+} \times \mathcal{R}^{-}\right), \\
\frac{1}{3} \text { otherwise. }
\end{array}\right. \\
& a_{2}\left(R_{1}, R_{3}\right)=\left\{\begin{array}{l}
\frac{1}{2} \text { if }\left(R_{1}, R_{3}\right) \in\left(\mathcal{R}^{+} \times \mathcal{R}^{-}\right), \\
\frac{1}{6} \text { if }\left(R_{1}, R_{3}\right) \in\left(\mathcal{R}^{-} \times \mathcal{R}^{+}\right), \\
\frac{1}{3} \text { otherwise. }
\end{array}\right. \\
& a_{3}\left(R_{1}, R_{2}\right)=\left\{\begin{array}{l}
\frac{1}{2} \text { if }\left(R_{1}, R_{2}\right) \in\left(\mathcal{R}^{-} \times \mathcal{R}^{+}\right), \\
\frac{1}{6} \text { if }\left(R_{1}, R_{2}\right) \in\left(\mathcal{R}^{+} \times \mathcal{R}^{-}\right), \\
\frac{1}{3} \text { otherwise. }
\end{array}\right.
\end{aligned}
$$

This function is efficient, strategy-proof, and satisfies uniform treatment of uniforms.

\section{References}

[1] S. Barberì and M. O. JaCkson, 'Strategy-Proof Exchange', Econometrica, 63 (1995), pp 51-87.

[2] S. Barberì, M. O. Jackson, And A. Neme, 'Strategy-Proof Allotment Rules', Working paper, Universitat Autónoma de Barcelona, 1995.

[3] J. P. Benassy, 'The Economics of Market Disequilibrium, Academic Press: New-York.

[4] S. Ching, 'An Alternative Characterization of the Uniform Rule', Social Choice and Welfare, 11 vol 2 (1994), pp 131-136.

[5] M. Satterthwaite and H. Sonnenschein, 'Strategyproof Allocation Mechanisms at Differentiable Points', Rev. Econ. Studies, 48 (1981), pp 587-597.

[6] S. SERIzawa, 'Strategy-Proof and Individually Rational Social Choice Functions for Public Good Economies', Econ. Theory, 7 (1996), pp $501-512$.

[7] S. SHenker, 'On the Strategy-Proof and Smooth Allocation of Private Goods in a Production Economy, mimeo, Xerox PARC, 1992. 
[8] Y. Sprumont, 'The Division Problem with Single-Peaked Preferences: a Characterization of the Uniform Allocation Rule', Econometrica, 59 (1991), pp 509-520.

[9] L. ZHou, 'Inefficiency of Strategy-Proof Allocation Mechanisms in Pure Exchange Economies', Soc. Choice Welfare, 8 (1991), pp 247-254. 